American Journal of Applied Sciences 9 (8): 1150-1157, 2012

ISSN 1546-9239

(C) 2012 Science Publications

\title{
A Reliable Identification System for Red Palm Weevil
}

\author{
Saleh Mufleh Al-Saqer \\ Department of Agricultural Engineering, \\ College of Food and Agriculture Sciences, \\ King Saud University, Riyadh, Kingdom of Saudi Arabia
}

\begin{abstract}
Problem statement: Red Palm Weevil (RPW) is a widely found pest among palm trees and is known to cause significant losses every year to palm growers. Existing identification techniques for RPW comprise of using traps with pheromones to detect these pests. However, these traditional methods are labor-intensive, expensive to implement and unreliable for early detection of RPW infestation. Early detection of these pests would provide the best opportunity to eradicate them and minimize the potential losses of palm trees. Approach: In this study, a reliable identification system is developed to identify RPW by using only a small number of image descriptors in combination with neural network models. The neural networks were developed by using between three to nine image descriptors as inputs and a large database of insects' images was used for training. Three different training ratios ranging from $25-75 \%$ were used and the network was trained by two different algorithms. Further, several scenarios were formulated to test the efficacy and reliability of the newly developed identification system. Results: The results indicate that the identification system developed in this study is capable of $100 \%$ recognition of RPW and $93 \%$ recognition of other insects in the database by taking as input only three easily-calculable image descriptors. Further, the average training times for these networks was $13 \mathrm{sec}$ and the testing time for a single image was only $0.015 \mathrm{sec}$. Conclusion: The new system developed in this study provided reliable identification for RPW and was found to be up to 14 times faster in training and three times faster in testing of insects' images.
\end{abstract}

Key words: Regional Properties (RP), Red Palm Weevil (RPW), Zernike Moments (ZM), Integrated Pest Management (IPM), Artificial Neural Networks (ANN)

\section{INTRODUCTION}

Red Palm Weevil (RPW), Rynchophorus Ferrugineous Olivier, was discovered in southern and southeastern Asia in the early 1900s. It is now considered as the most lethal pest affecting palm trees and its existence has been reported all over the world (Abraham et al., 1998; Buxton, 1920; CISR, 2011; Faleiro, 2006; Lefroy, 1907; Li et al., 2009).

RPW attacks the palm tree and feeds on its tissues and remains inside the tree for generations protected and unnoticed from outside. This behavior explains the significance of this insect and the challenge of its monitoring and controlling (Esteban-Duran et al., 1998; Faleiro, 2006; Murphy and Briscoe, 1999). It emerges out of the infested palm tree when the tree is hollow from inside to target another host. Currently, the infested palm trees are burnt to save other palm trees and control the spread of the RPW.

Numerous techniques to control and monitor RPW have been proposed. The Integrated Pest Management (IPM) developed by (Abraham et al., 1989) produced the best results. IPM is a multidimensional strategy which includes prevention and control techniques in addition to educational efforts. One major component of IPM is the early detection and trapping of RPW, which was reported by (Faleiro, 2006) to be a critical aspect of this procedure. Thus, major efforts are placed to improve this technique. The idea is to determine the possible existence of RPWs before they attack palm trees and to protect the uninfected area. The trapping consists of spreading out traps in the entire region which contains bait, pheromone and pesticide. The recommended density of traps is reported to be 1-2 traps per hectare (Faleiro, 2006; Soroker et al., 2005). These traps are inspected and maintained on a regular basis, which is laborious and a time-consuming task.

The automation of the inspection process for the traps may be achieved by using wireless image sensor network which may also help to detect early existence of RPW efficiently. The core idea is to capture an image of an insect in a trap and identify the existence of the RPW. All motes (nodes) of wireless image sensor network coordinate with each other and forward the corresponding information to the main server. The use of 
wireless sensor network is already established in fields such as poultry (Murad et al., 2009), steel industry (Jan et al., 2010) and agriculture (Burrell et al., 2004). The use of wireless image sensor network has been adopted in several fields for object detection and recognition (Kulkarni et al., 2005), fruit flies surveillance (Liu et al., 2009), environment observation and surveillance (Feng et al., 2005).

Reliability and processing time plays a critical role in the success of any recognition system. Few insect recognition and identification systems have been proposed such as Automated Insect Identification through Concatenated Histograms of Local Appearance System (AIICHLA) for the identification of Stonefly larvae (Larios et al., 2007); Automated Bee Identification System (ABIS) for the identification of bees (Arbuckle et al., 2001); Species Identification Automated and Web Accessible System (SPIWA) for identification of spiders (Do et al., 1999); software system developed for the identification of Pecan Weevil (Ashaghathra, 2008); Digital Automated Identification System (DAISY) for the Pecan Weevilon of Ophioninae (Watson et al., 2004 ).

Artificial Neural Networks (ANN) are a powerful method for solving highly non-linear, complex pattern recognition problems. Using the ANN, Do et al. (1999) developed the SPIWA system for the identification of Spiders, Balfoort et al. (1992) developed the AAIS system for identification of algae, France et al. (2000) proposed the PICS system for identification of Pollen, Lin et al. (1997) developed the Face Recognition System, Gutta et al. (2000) developed the human classification system for identification of gender, ethnicity and human face pose.

The fundamental aspect of ANN is training (learning) processed, where the network is exposed to inputs and/or outputs and the training algorithm is used to update the network parameters to achieve optimal solution. In 1986, the multi-layer perceptron based on the back-propagation algorithm was developed for training multilayer ANN. This development is largely attributed with revolutionizing the field of ANN by making the solution of large scale problems possible (Rumelhart et al., 1986). Since that development, the applications of multilayer neural networks have grown steadily in diverse fields.

In this study, multilayer neural networks were developed to identify RPW among other insects. The ultimate goal of this research is to develop an early detection automated system for identifying RPW in the field. The first phase of automating the inspection process is to develop an identification system and necessary software that would recognize RPW based on machine vision. In our previous studies, an RPW recognition system was proposed using two different image processing techniques (Al-Saqer and Hassan, 2011b). The system utilized standard image processing techniques and implemented the template matching method for identifying red palm weevil. Using that system, the processing time was found to be about 0.47 sec. While the success rate for identification of the RPW and other insects was 97 and $88 \%$, respectively. In another study, a system based on the Support Vector Machine (SVM) method that utilizes descriptors derived from standard image processing techniques was used for recognition of RPW (Hassan and Al-Saqer, 2012). Recently, a neural network-based system was developed that utilized the binary images (pixel data) directly to identify RPW (Al-Saqer and Hassan, 2011a). However, this method was computationally expensive for practical field applications. In particular, the testing times per image were relatively large and the memory requirements for storing the binary images were prohibitive for portable, field applications. Therefore, in this study, a new approach is presented such that only limited input information is necessary to the neural networks for positive identification of the RPW. The new approach comprises of utilizing as input the image descriptors obtained from image processing techniques rather than the binary pixel data directly. As will be shown later in this contribution, using as little as three easily-calculable image descriptors provided reliable recognition of RPW with a diverse database of insects' images. The approach presented in this study is a departure from our previous study (Al-Saqer and Hassan, 2011a) in identifying RPW and requires significantly less time both for training and testing of the neural networks.

\section{MATERIALS AND METHODS}

Image acquisition: In object recognition applications, the size and variation in training data play a pivotal role. In this study, a wide range of samples of the RPW and other insects were collected (Table 1). The images of these insects were captured by a Sony Cyber-Shot DSC-HX1 camera which can capture 10 frames per sec at 9.1 megapixel resolution and $20 \times$ optimal zoom.

The acquired images were $3456 \times 2592$ pixels and these images were converted to binary format and resized to $501 \times 519$ pixels. A Dell OptiPlex 780 PC equipped with Intel Core 2 Duo E8400 $3.0 \mathrm{GHz}$ processor and 4 GB RAM was used in this study. The software used was MATLAB ${ }^{\circledR}$ v 7.9.0.529 (R2006a). 
Table 1:Other Insects used in the database for developing ANN for RPW recognition system

Scientific name (Family/order)

Calosoma Chlorostictum (Carabidae/Coleoptera)

Gryllus Bimaculatus (Gryllidae/Orthoptera)

Conocephalus Conocephalus (Tettigoniidae/Orthoptera)

Gryllotalpa Gryllotalpa (Gryllotalpidae/Orthoptera)

Gryllotalpa Africana (Gryllotalpidae/Orthoptera)

Oryctes Nasicornis (Scarabaeidae/Coleoptera)

Cybister Tripunctatus Africana (Dytiscidae/Coleoptera)

Scarites Eurytus (Carabidae/Coleoptera)

Lanelater Motodenta (Elateroidea/Coleoptera)

Mlabri Tenebrosa (Meloidae/Coleoptera)

Hyles Lineata Livornica (Sphingidae/Lepidoptera)

Coccotrypes advena (Curculionidae/Coleoptera)

Gnopholeon sp. (Myrmeleontidae/Neuroptera)

Blepharopsis Mindica (Mantidae/Mantodea)

Anax sp. (Aeshnidae/Odonata)

Xylocopa Hottentota (Anthophoridae/Hymenoptera)

Poikiloderma (Pamphiliidae/Orthoptera)

Lophyra sp. (Carabidae/Coleoptera)

Scarabaens sp. (Scarabaeidae/Coleoptera)

Cerceris Rybyensis (Sphecidae/Hymenoptra)

Data processing method: Two standard image processing techniques were used to obtain the descriptor that will serve as inputs for developing the ANN. The two standard techniques were the Regional Properties and Zernike Moments. In the Regional Properties technique, three descriptors derived from the binary image of the insects were obtained. These descriptors were the lengths of major and minor axes of the region and area of the region. The value of the area is determined by counting the number of pixels connected with each other in the image. Similarly, the lengths of major and minor axes are obtained by counting the pixels in the major and minor axes of the elliptical region in the image (Gonzalez and Woods, 2002). The calculated values of major and minor axes as well as the normalized value of the area were used as input to the ANN.

The second method, Zernike Moments, involves introduction of a set of complex polynomials which formulate an orthogonal set over the interior of an object's circle. The center of the circle is considered as the origin and pixel coordinates are mapped to a unit circle. Any pixel found outside the circle is not considered in the computation of Zernike Moments. Due to orthogonal properties, overlapping and repetition of information among moments with different orders is not possible. Hence, representation of an image is unique and independent for each moment (Whoi-Yul and Yong-Sung, 2000). Zernike Moments of the third order were used in this study and the six values of the moments were used as inputs to the ANN. These two image processing techniques are utilized in image recognition applications due to their rotation invariance, expression efficiency and noise robustness.
Artificial neural networks: The development of ANN or Neural Network (NN) is inspired by the working of the human brain. This non-linear data modeling technique is capable of approximating complex relationships between input and output and has been used for several pattern recognition applications (Bishop, 1996). A single neuron in ANN can be represented mathematically as Eq. 1:

$y=f\left(\sum_{i=j}^{n} w_{i} x_{i}+b\right)$

where, $\mathrm{x}$ is input, $\mathrm{w}$ is the weight and $\mathrm{b}$ is the bias of $\mathrm{a}$ neuron. The output $\mathrm{y}$ is dependent on the inputs, weights, bias and the transfer function, f, which is generally a sigmoidal function.

ANN is categorized in three modes on the basis of training methods used: supervised, unsupervised and reinforcement. Pattern recognition typically uses supervised training where inputs and outputs are provided to ANN for learning purposes. The efficiency of trained ANN is dependent on the scale and variety of training data.

ANN derives their powerful pattern recognition capabilities of the complex network architecture contained in them. Such network architecture comprises of the number of hidden layers and neurons for a typical feed-forward multilayer perceptron based on the backpropagation algorithm (Hagan et al., 2002). Although an optimal network architecture is a trial and error procedure and is problem-dependent, certain simple guidelines have been presented recently to evaluate the number of hidden neurons to be used in a network layer. $\mathrm{Xu}$ and Chen (2008) reported that the optimal number of neurons ' $\mathrm{n}$ ' in a hidden layer is dependent on the dimension of the input ' $\mathrm{d}$ ' and number of training pairs ' $\mathrm{N}$ ' for small or medium size datasets i.e., $n=N / d$ if its value is below or close to 30 ; else $n=\left(\frac{N}{d \log N}\right)^{1 / 2}$. They categorized the training dataset to be medium or small if training pairs are less than 5000. Note that this method does not consider the problem of local minima ( $\mathrm{Xu}$ and Chen, 2008). In this work, the method proposed by $\mathrm{Xu}$ and Chen (2008) was adopted for the number of hidden neurons in a single hidden-layer neural networks. To avoid local minima, the neural networks developed in this study were trained 20 times for each case by using gradient-based search algorithms in combination with the back-propagation updates of the network parameters (weights and biases). In particular, the Scaled Conjugate Gradient Algorithm (SCG) and Conjugate Gradient with Powell/Beale 
Restart Algorithm (CGB) were selected since they have been found useful in pattern recognition problems (AlSaqer and Hassan, 2011a; Beale et al., 2010; Johansson et al., 1991; Moller, 1993; Powell, 1977). At the end of simulations, the best 20 networks were selected for further analysis. Further, in all cases, an early stopping criterion was used to prevent over-fitting of the data by the neural network (Hagan et al., 2002). All the transfer functions used in the network were sigmoidal functions.

The number of images used for training and testing were 419, which included 326 images of RPW and 93 images of other insects that are normally found in the habitat of palm trees. Three different training ratios of 25,50 and $75 \%$ were used for training the neural networks while the remaining data were used for testing the network. Further, the training set was randomly divided 10 times for each of the 20 trials of the network. In this manner, a total of 200 networks was generated for further analysis. Three different scenarios were considered for the network inputs. The inputs to ANN were provided from the descriptors obtained by the Zernike Moments (ZM), Regional Properties (RP) and a combination of both RP and ZM (RPZM). Thus, the number of inputs in the networks were 3,6 or a total of 9 image descriptors.

Error in classification: In this study, the error is described as wrong classification of RPW to be another insect or vice versa. Consequently, this error can be categorized into two types i.e., Type-I and Type-II, where the first one refers to the misclassification of other insects while the second type is misclassification of the RPW. Evidently, Type-II error is more critical in this study when compared to Type-I error. Overall, the system's sensitivity to the identification of RPW may be described as Type-I error, while Type-II error may be viewed as the inefficiency of the system.

\section{RESULTS AND DISCUSSION}

In this study, artificial neural networks were used to identify RPW using key image descriptors as inputs. Several scenarios were considered to investigate the efficacy and reliability of the neural networks in predicting RPW correctly. The following three scenarios were considered:

- Three descriptors from regional properties were used as network inputs

- Six descriptors from Zernike moments were used as network inputs

- Nine descriptors (from scenarios 1 and 2) were used as network inputs
Further, three different training ratios were used to test the network's performance and robustness. In particular, 25, 50 and $75 \%$ of the data were used for training purposes and in each case two different training algorithms (SCG and CGB) were used to test the sensitivity of neural networks to the training algorithms used.

Table 2 lists the summary results obtained for the neural networks developed in this study. The table also compares the results obtained in this study with earlier works where template matching (Al-Saqer and Hassan, 2011b), support vector machine (Hassan and Al-Saqer, 2012) and ANN with binary images (Al-Saqer and Hassan, 2011a) were used to identify RPW. As evident from Table 2, the current study provides the best results in terms of recognition of the RPW and processing times. Note that these results were obtained using only the descriptors obtained from regional properties and Zernike moments. In an earlier study (Al-Saqer and Hassan, 2011a), binary images were directly used to train the neural networks. However, the older scheme was computationally expensive as seen from the training times in Table 2. Further, the neural networks developed in that study (Al-Saqer and Hassan, 2011a) had very large memory requirements, which is a significant disadvantage for portable, wireless-based field applications. In contrast, the networks developed in this study require only three to nine image descriptors for reliable identification of the RPW. Thus, the networks developed in the current study are ideally suited as part of an image recognition system for field applications. Note that the support vector machine-based identification system developed earlier (Hassan and AlSaqer, 2012) exhibits similar performance (albeit, slightly lower recognition rates for RPW) than the neural networks developed in this study, as shown in Table 2.

In each case, the neural networks were trained 20 times and the best network performance as well as the average of all the trials was analyzed to investigate the sensitivity of the results of the training algorithm used as well as the effect of training ratios and the number of descriptors used. Figures 1 and 2 show the best and average trained networks, respectively, obtained with the SCG algorithm. Note that the figures present the results in terms of Type I and II errors in each case. As shown in these figures, when only three descriptors are used for the Regional Properties (RP), the recognition rates for RPW and other insects are 99 and $90 \%$, respectively, when the training ratio is $25 \%$. 
Am. J. Applied Sci., 9 (8): 1150-1157, 2012

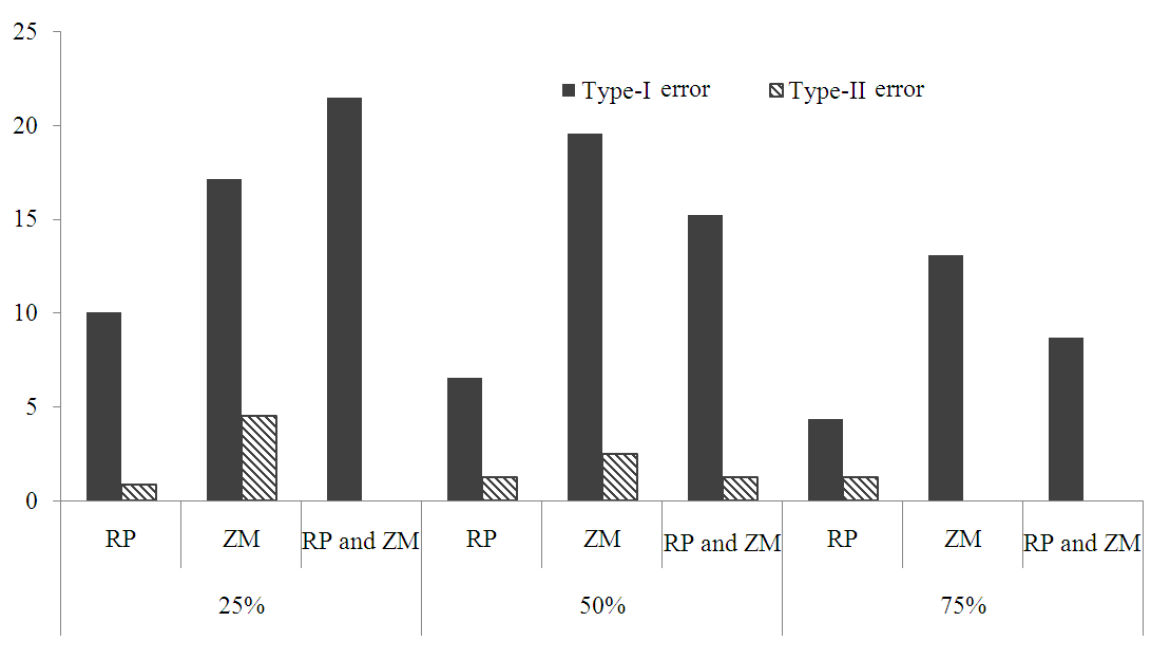

Fig. 1: Best recognition results for RPW obtained using the Scaled Conjugate Gradient (SCG) algorithm

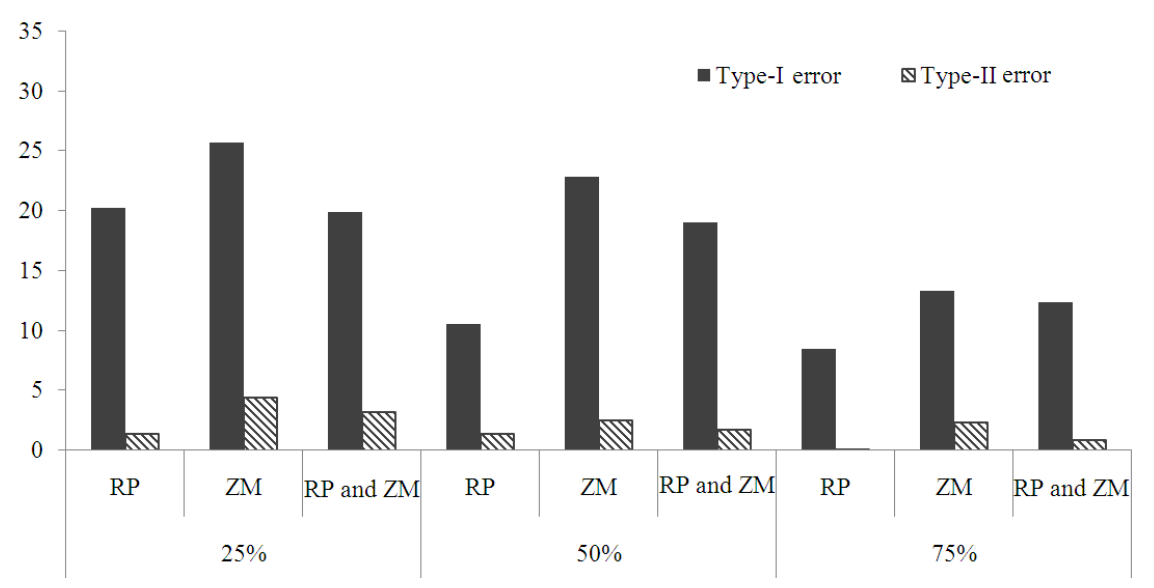

Fig. 2: Average recognition results for RPW obtained using the Scaled Conjugate Gradient (SCG) algorithm

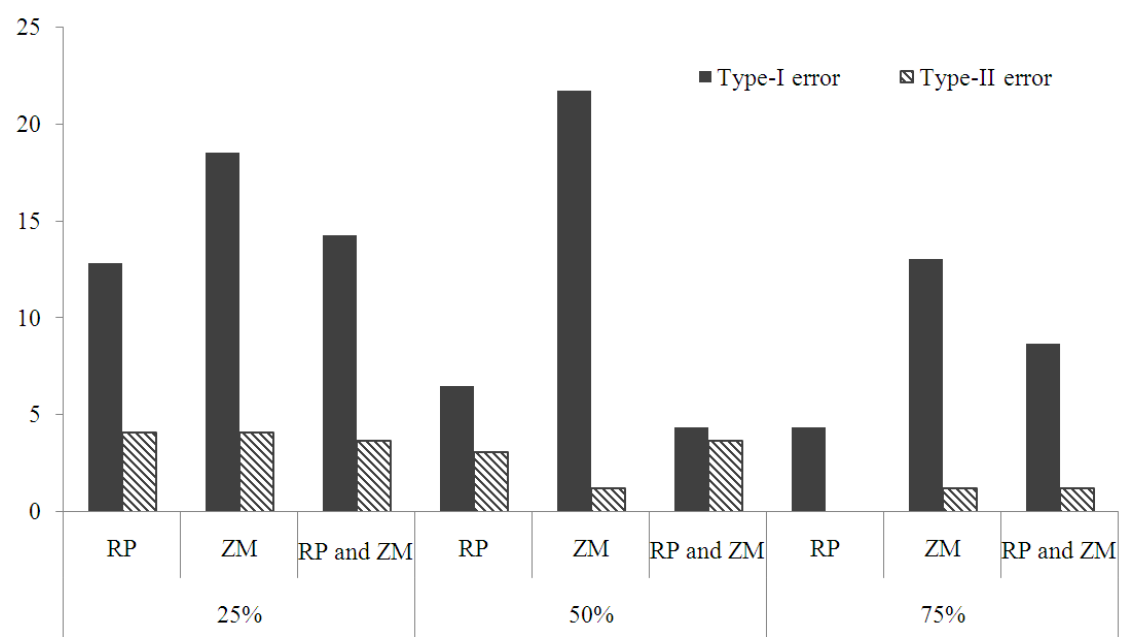

Fig. 3: Best recognition results for RPW obtained using the Conjugate Gradient Powell/Beale (CGB) algorithm 1154 
Am. J. Applied Sci., 9 (8): 1150-1157, 2012

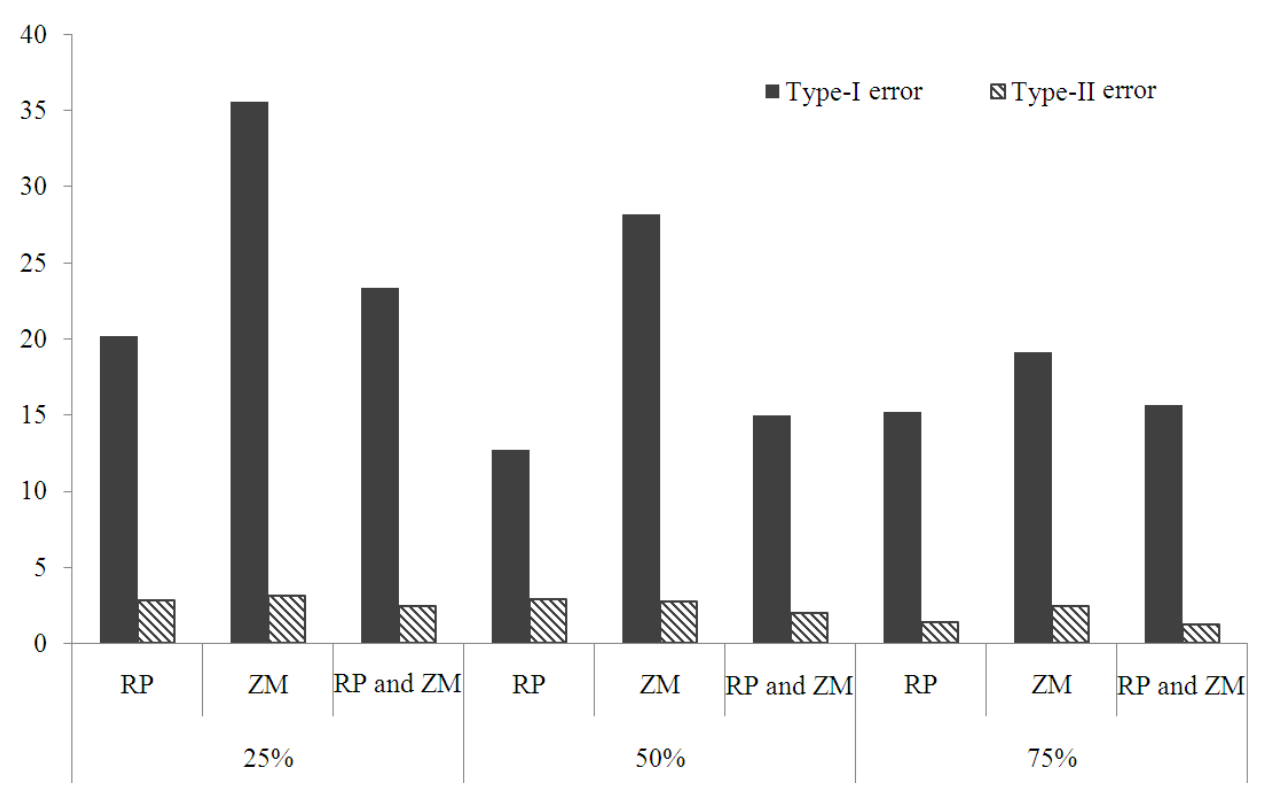

Fig. 4: Average recognition results for RPW obtained using the Conjugate Gradient Powell/Beale (CGB) algorithm

Table 2: Summary results in recognition of RPW with different techniques

\begin{tabular}{|c|c|c|c|c|c|}
\hline \multirow[b]{2}{*}{ Technique } & \multicolumn{2}{|c|}{ Recognition rate* } & \multicolumn{2}{|c|}{ Processing time $(\mathrm{sec})$} & \multirow[b]{2}{*}{ Source } \\
\hline & RPW & Other insects & Training & Testing & \\
\hline Template Matching & 97 & 88 & - & 0.47 & $\begin{array}{l}\text { (Al-Saqer and } \\
\text { Hassan, 2011b) }\end{array}$ \\
\hline $\begin{array}{l}\text { ANN (binary image } \\
\text { data as input) }\end{array}$ & 99 & 93 & 183.4 & 0.042 & $\begin{array}{l}\text { (Al-Saqer and } \\
\text { Hassan, 2011a) }\end{array}$ \\
\hline $\begin{array}{l}\text { SVM (image } \\
\text { descriptors as input) }\end{array}$ & 97 & 93 & 10.5 & 0.015 & $\begin{array}{l}\text { (Hassan and } \\
\text { Al-Saqer, 2012) }\end{array}$ \\
\hline $\begin{array}{l}\text { ANN (image } \\
\text { descriptors as input) }\end{array}$ & 100 & 93 & 13 & 0.015 & This Study \\
\hline
\end{tabular}

Recognition rates are in percentages

Further, the corresponding statistics for recognition were 99 and $96 \%$ when the training ratio was increased to $75 \%$. Thus, as expected, the recognition results improve overall when the training ratio was increased. Further, the average network performance in Fig. 2 also shows similar trend and behavior.

Figures 3 and 4 show the corresponding results for CGB algorithm. Similar to the case with the SCG algorithm, the recognition results increase with the increase in training ratio and RP descriptors provide the best overall results with the least amount of input information. In particular, the highest recognition rates of 100 and $96 \%$ were obtained when using the RP descriptors alone and $75 \%$ of the data as training set. Thus, these results are consistent with the results obtained from the SCG algorithm, indicating that either of these algorithms is capable of providing optimal training for the neural networks in this study. Further, as shown in Table 2, the neural networks developed in this study are computationally more efficient and require the least amount of information as inputs compared to previous studies. The set of information required can be derived from the three regional properties, namely, the length of major and minor axes and the area of the image. Thus, the results reported in this study appear to be promising new developments in recognizing RPW and would be helpful in developing compact, efficient and robust wireless image sensor network that utilizes the recognition capabilities developed in this study.

\section{CONCLUSION}

This research was a continuous effort of our earlier work to develop reliable and efficient identification systems for RPW. In this study, up to nine easilycalculable image descriptors were used as inputs to artificial neural networks. The descriptors were derived from standard image processing techniques, namely, Regional Properties (RP) and Zernike Moments (ZM). The neural networks were trained with different training ratios $(25-75 \%)$ of the total data set that comprised of 326 RPW and 93 other insects' images. For each training ratio, the networks were trained by two different training algorithms-Scaled Conjugate Gradient (SCG) and Conjugate Gradient with Powell/Beale restarts (CGB). In each case, the network was trained 20 times to avoid convergence to local minima and the training ratio was randomly selected 10 times.

Results indicate that the neural networks developed in this study are capable of $100 \%$ recognition rates for RPW and 93\% for other insects' images when only 
three descriptors originating from the RP method are used and $75 \%$ of the data set is used for training purposes. The results of both algorithms were comparable in detecting the RPW and either algorithm may be used to train the networks efficiently. The average training times were about $13 \mathrm{sec}$ and the testing time for a single image was only $0.015 \mathrm{sec}$. The neural networks developed in this study require up to 14 times less training time and were three times faster in testing for a single image. The networks developed in this study appear to be reliable and efficient and would prove helpful in developing wireless sensor network for field applications.

\section{ACKNOWLEDGMENT}

The financial support of the Research Center of College of Food and Agriculture Sciences, Deanship of Scientific Research, King Saud University is gratefully acknowledged. The author is very thankful to Dr. Sayeed Mohammed Ahmed for his support.

\section{REFERENCES}

Abraham, V.A., K.M.A. Koya and C. Kurian, 1989. Integrated management of red palm weevil (Rhynchophorus Ferrugineus O.) in coconut gardens. J. Plantation Crops 16: 159-162.

Abraham, V.A., M. Al-Shuaibi, J.R. Faleiro, R.A. Abozuhairah and P.S.P.V. Vidyasagar, 1998. An integrated approach for the management of red palm weevil Rhynchophorus ferrugineus Olivier-A key pest of date palm in the Middle East. J. Sci. Res. Agric. Sci., 3: 77-83.

Al-Saqer, S.M. and G.M. Hassan, 2011a. Artificial neural networks based red palm weevil (Rynchophorus Ferrugineous, Olivier) recognition system. Am. J. Agric. Biol. Sci., 6: 356-364. DOI: 10.3844/ajabssp. 2011.356.364

Al-Saqer, S.M. and G.M. Hassan, 2011b. Red palm weevil (Rynchophorus Ferrugineous, Olivier) recognition by image processing techniques. Am. J. Agric. Biol. Sci., 6: 365-376. DOI: 10.3844/ajabssp. 2011.365.376

Arbuckle, T., S. Schroder, V. Steinhage and D. Wittmann, 2001. Biodiversity informatics in action: Identification and monitoring of bee species using ABIS. Proceedings of the 15th International Symposium on Informatics for Environmental Protection, (IEP' 01), Zurich, pp: 425-430.

Ashaghathra, S.M., 2008. Identification of Pecan Weevils Through Image Processing. 1st Edn., ProQuest, ISBN-10: 0549560947, pp: 148.
Balfoort, H.W., J. Snoek, J.R.M. Smiths, L.W. Breedveld and J.W. Hofstraat et al., 1992. Automatic Identification of algae: Neural network analysis of flow cytometric data. J. Plankton Res., 14: 575-589. DOI: 10.1093/plankt/14.4.575

Beale, M.H., M.T. Hagan and H.B. Demuth, 2010. Neural network toolbox ${ }^{\text {TM }} 7$ user's guide. The Math Works, Inc.

Bishop, C.M., 1996. Neural Networks for Pattern Recognition. 1st Edn., Oxford University Press, USA, ISBN-10: 0198538642, pp: 482.

Burrell, J., T. Brooke and R. Beckwith, 2004. Vineyard computing: Sensor networks in agricultural production. IEEE Pervasive Comput., 3: 38-45. DOI: 10.1109/MPRV.2004.1269130

Buxton, P.A., 1920. Insect pests of dates and the date palm in Mesopotamia and elsewhere. Bull. Entomol. Res., 11: 287-304. DOI: 10.1017/S0007485300044709

CISR, 2011. Red Palm Weevil, Rhynchophorus ferrugineus (olivier) (coleopetera: Curculionidae), center of invasive species research. Mike Lewis, CISR.

Do, M.T., J.M. Harp and K.C. Norris, 1999. A test of a pattern recognition system for identification of Spiders. Bull. Entomol. Res., 89: 217-224. DOI: 10.1017/S0007485399000334

Esteban-Duran, J.E., J.L. Yela, F.B. Crespo and A.J. Alvarez, 1998. Biology of red palm weevil, Rhynchophorus ferrugineus (Olivier) Coleoptera: Curculionidae: Rhynchophorinae, in the laboratory and field, life cycle, biological characteristics in its zone of introduction in Spain, biological method of detection and possible control. Boletin Sanidad Vegetal Plagas 24: 737-748.

Faleiro, J.R., 2006. A review of the issues and management of the red palm weevil Rhynchophorus ferrugineus (Coleoptera: Rhynchophoridae) in coconut and date palm during the last one hundred years. Int. J. Tropical Insect Sci., 26: 135-154. DOI: 10.1079/IJT2006113

Feng, W.C., E. Kaiser and M.L. Baillif, 2005. Panoptes: Scalable low-power video sensor networking technologies. ACM Trans. Multimedia Comput. Commun. Appli., 1: 151-167. DOI: 10.1145/1062253.1062256

France, I., A.W.G. Duller, G.A.T. Duller and H.F. Lamb, 2000. A new approach to automated pollen analysis. Quaternary Sci. Rev., 19: 537-546. DOI: 10.1016/S0277-3791(99)00021-9

Gonzalez, R.C. and R.E. Woods, 2002. Digital Image Processing, 2nd Edn., Prentice Hall, Upper Saddle River, New Jersey. 
Gutta, S., J.R.J. Huang, P. Jonathon and H. Wechsler, 2000. Mixture of experts for classification of gender, ethnic origin and pose of human faces. IEEE Trans. Neural Netw., 11: 948-960. DOI: 10.1109/72.857774

Hagan, M.T., H.B. Demuth and M.H. Beale, 2002. Neural Network Design. 1st Edn., PWS Publishing, Boston, ISBN-10: 0534943322, pp: 736.

Hassan, G.M. and S.M. Al-Saqer, 2012. Support Vector Machine based red palm weevil (Rynchophorus Ferrugineous, Olivier). Recognition Syst. Am. J. Agric. Biol. Sci., 7: 36-42. DOI: 10.3844/ajabssp.2012.36.42

Jan, M.F., Q. Habib, M. Irfan, M. Murad and K.M. Yahya et al., 2010. Carbon monoxide detection and autonomous countermeasure system for a steel mill using wireless sensor and actuator network. Proceedings of the IEEE 6th International Conference on Emerging Technologies, Oct. 1819, Islamabad, pp: 405-409. DOI: 10.1109/ICET.2010.5638502

Johansson, E.M, F.U. Dowla and D.M. Goodman, 1991. Backpropagation learning for multilayer feed-forward neural networks using the conjugate gradient method. Int. J. Neural Syst., 2: 291-301. DOI: $10.1142 / \mathrm{S} 0129065791000261$

Whoi-Yul, K. and K. Yong-Sung, 2000. A region-based shape descriptor using Zernike moments. Signal Process: Image Commun., 16: 95-102. DOI: 10.1016/S0923-5965 (00)00019-9

Kulkarni, P., D. Ganesan, P. Shenoy and Q. Lu, 2005. Sense Eye: A multi-tier camera sensor network. Proceedings of the 13th Annual ACM International Conference on Multimedia, Nov. 06-12, Singapore, pp: 229-238.

Larios, N., H. Deng, W. Zhang, M. Sarpola and J. Yuen et al., 2007. Automated insect identification through concatenated histograms of local appearance features. Proceedings of the IEEE Workshop on Applications of Computer Vision (WACV' 07), IEEE Xplore Press, Austin, TX, pp: 26-26. DOI: 10.1109/WACV.2007.13

Lefroy, H.M., 1907. The more important insects injurious to Indian agriculture memoirs of the department of agriculture in India. Nature, 76: 588588. DOI: $10.1038 / 076588 \mathrm{a} 0$

Li, Y., Z.R. Zhu, R. Ju and L.S. Wang, 2009. The red palm weevil, Rhynchophorus ferrugineus (Coleoptera: Curculionidae), newly reported from Zhejiang, China and update of geographical distribution. Florida Entomol., 92: 386-387. DOI: 10.1653/024.092.0229
Lin, S.H., S.Y. Kung and L.J. Lin, 1997. Face recognition/detection by probabilistic decision based neural network. IEEE Trans. Neural Netw., 8: 114-132. DOI: $10.1109 / 72.554196$

Liu, Y., J. Zhang, M. Richards, B. Pham and P. Roe, 2009. Towards continuous surveillance of fruit flies using sensor networks and machine vision. Proceedings of the 5th International Conference on Wireless Communications Networking and Mobile Computing, Sept. 24-26, Beijing, pp: 4705-4709.

Moller, M., 1993. A scaled conjugate gradient algorithm for fast supervised learning. J. Neural Netw., 6: 525-533. DOI: 10.1016/S0893-6080 (05)80056-5

Murad, M., K.M. Yahya and G.M. Hassan, 2009. Web based poultry farm monitoring system using wireless sensor network. Proceedings of the 7 th International Conference on Frontiers of Information Technology, Dec. 16-18, Pakistan. DOI: $10.1145 / 1838002.1838010$

Murphy, S.T. and B.R. Briscoe, 1999. The red palm weevil as an alien invasive: Biology and the prospects for Biological Control as a component of IPM. Biocontrol News Inform. 20: 35N-45N.

Powell, M.J.D., 1977. Restart procedures for the conjugate gradient method. J. Math. Program., 12: 241-254.DOI: $10.1007 / \mathrm{BF} 01593790$

Rumelhart, D., G. Hinton and R. Williams, 1986. Learning representations by back-propagating errors. Lett. Nat., 323: 533-536. DOI: 10.1038/323533a0

Soroker, V., D. Blumberg, A. Haberman, M. Hamburger-Rishad and S. Reneh, 2005. Current status of red palm weevil infestation in date palm plantations in Israel. Phytoparasitica, 33: 97-106. DOI: $10.1007 / \mathrm{BF} 02980931$

$\mathrm{Xu}, \mathrm{S}$. and L. Chen, 2008. A novel approach for determining the optimal number of hidden layer neurons for FNN's and its application in data mining. Proceedings of the 5th International Conference on Information Technology and Applications, Queensland, Australia, Jun. 23-26. pp: 683-689. 